\title{
Andrew Lenard: A Mystery Unraveled
}

\author{
Jeffery PRAUGHT and Roman G. SMIRNOV* \\ Department of Mathematics and Statistics, Dalhousie University, \\ Halifax, Nova Scotia, Canada, B3H 3J5 \\ E-mail: praughtj@mathstat.dal.ca,smirnov@mathstat.dal.ca \\ * URL: http://www.mathstat.dal.ca/ smirnov/
}

Received September 29, 2005, in final form October 03, 2005; Published online October 08, 2005

Original article is available at http://www.emis.de/journals/SIGMA/2005/Paper005/

\begin{abstract}
The theory of bi-Hamiltonian systems has its roots in what is commonly referred to as the "Lenard recursion formula". The story about the discovery of the formula told by Andrew Lenard is the subject of this article.

Key words: Lenard's recursion formula; bi-Hamiltonian formalism; Korteweg-de Vries equation

2000 Mathematics Subject Classification: 01A60; 35Q53; 35Q51; 70H06
\end{abstract}

\section{Introduction}

This aim of this review article is to present the untold story about the use of the name Lenard in many concepts that form the backbone of bi-Hamiltonian (multi-Hamiltonian) theory. Originally, the theory came to prominence with the fundamental 1978 paper by Franco Magri [17], followed almost immediately by the 1979 paper due to Israel Gel'fand and Irina Dorfman [13] that developed and extended the results presented in [16] and [17]. Since then, many scientists have been working on the development of the theory of bi-Hamiltonian systems, making it one of the most active areas of research in the field of mathematical physics (see, for example, $[1,2,3,4,5,6,8,9,13,14,17,18,19,20,24,25,26]$, as well as the relevant references therein).

The majority of the hundreds of papers written to date on the subject are invariably based on the use of such concepts as the "Lenard bicomplex", "Lenard chain", "Lenard recursion operator", "Lenard scheme", and so forth (see, for instance, $[1,14,18,20]$ ). This leads one to believe that Andrew Lenard must have made a fundamental contribution to the theory, yet, as everybody working in the area knows, no paper on the subject under his name has ever been written. Although the papers by Clifford Gardner et al [12] and Peter Lax [16] contain short paragraphs that strongly allude to Andrew Lenard's contribution, the whole story told by him (see below) appears to be as fascinating as the result itself. Furthermore, it must be said that the discovery of Lenard had fundamental consequences beyond the theory of bi-Hamiltonian systems that it originated. The notion of a recursion operator, introduced by Peter Olver in [23], is not, in general, a byproduct of the existence of two or more Hamiltonian structures. Although the Lenard recursion operator for the Korteweg-de Vries equation comes from the recursion relation (15), more generally, a recursion operator is a property of a symmetry group nature (see $[23,24]$ for more details), rather than the existence of a bi-Hamiltonian structure.

In what follows, we reproduce ${ }^{1}$ the story obtained by one of us (JP) in full, preceded by a brief review of the mathematical background involved. We believe that the results of this historical investigation will be of interest to the scientists working in the area as well as anyone interested in the history of 20 th century mathematics.

\footnotetext{
${ }^{1}$ With A. Lenard's permission.
} 


\section{The emergence of the theory}

In the last forty years or so, the Korteweg-de Vries (KdV) equation has received much attention in the mathematical physics literature following the pioneering work of Kruskal and Zabusky [28] in the mid-sixties. As is well-known, in this work the authors have reported numerical observations demonstrating that the KdV solitary waves pass through each other with no change in shape or speed. The results presented in a series of papers by Gardner, Green, Kruskal, Miura and those that followed $[10,22,21,27,11,7,15,12]$, gave rise to the new theory of solitons and indicated applications to many areas of mathematical physics that are still being actively advanced today. Thus, for example, soon after the breakthrough of 1965, Gardner, Green, Kruskal, and Miura [10] enriched the theory with another fundamental development; it was a new method later called the inverse scattering method (ISM). In a nutshell, the method allows one to find the solution to the nonlinear problem of solving the KdV equation via a series of linear computations. Moreover, by using the ISM, as well as the new technique later called the Miura transform, the authors demonstrated the existence of an infinite number of conservation laws for solutions of the KdV equation and explicitly derived several of them [21, 22]. In turn, the existence of an infinite number of conserved quantities for the KdV equation was another important step in advancing the new theory.

Importantly, this discovery provided a framework for the introduction of a new Hamiltonian formalism, suitable not only for studying the KdV equation but also other nonlinear partial differential equations that exhibited similar properties (see [6] and the references therein). It was first shown that the Hamiltonian formalism of classical mechanics could be naturally incorporated into the study of the $\mathrm{KdV}$ equation. The consequences of this discovery, reported in 1971, independently and almost simultaneously by Gardner [11] and Faddeev \& Zakharov [7], eventually reached far beyond the study of the KdV equation. In what follows we reproduce the main features of the Hamiltonian formalism for the KdV equation and then show how the Lenard recursion formula fits naturally within the general theory.

Consider the $\mathrm{KdV}$ equation of the following form:

$$
u_{t}=6 u u_{x}+u_{x x x} .
$$

Gardner [11] and Faddeev \& Zakharov [7] observed that the right hand side of equation (1) can be rewritten as follows:

$$
u_{t}=P_{0} \frac{\delta H_{0}}{\delta u(x)},
$$

where $H_{0}$ is given by

$$
H_{0}=\int\left(u^{3}-\frac{1}{2} u_{x}^{2}\right) d x
$$

and

$$
P_{0}=\frac{\partial}{\partial x}
$$

while the expression $\frac{\delta}{\delta u(x)}$ denotes the gradient (or, the Frèchet derivative). The gradient $\delta_{u}$ acts on any functional $F=F\left(u, u_{x}, u_{x x}, \ldots\right)$ as follows:

$$
\frac{\delta F}{\delta u}=\frac{\partial f}{\partial u}-\frac{\partial}{\partial x}\left(\frac{\partial f}{\partial u_{x}}\right)+\frac{\partial^{2}}{\partial x^{2}}\left(\frac{\partial f}{\partial u_{x x}}\right)-\cdots
$$

where $F\left(u, u_{x}, u_{x x}, \ldots\right)=\int f\left(u, u_{x}, u_{x x}, \ldots\right) \mathrm{d} x$. 
One immediately observes that formula (2) bears a striking resemblance to the corresponding formula for a Hamiltonian vector field $X_{H_{0}}$ in classical mechanics, namely

$$
X_{H}=P_{0} \mathrm{~d} H_{0}
$$

or, alternatively, $X_{H}=\left[P_{0}, H_{0}\right]$, where $[\cdot, \cdot]$ denotes the Schouten bracket. The quantities $P_{0}$ and $H_{0}$ are the corresponding Poisson bi-vector and Hamiltonian function, respectively, defined on a finite-dimensional manifold $M$. The triple $\left(M, P_{0}, X_{H_{0}}\right)$ is said to be a Hamiltonian system. The most important feature of the Poission bi-vector that appears in formula (6) is that it can be used to define the corresponding Poisson bracket, which is the mapping $\{\cdot, \cdot\}_{0}: \mathcal{F}(M) \times \mathcal{F}(M)$ $\rightarrow \mathcal{F}(M)$ given by

$$
\left\{f_{1}, f_{2}\right\}_{0}:=P_{0} \mathrm{~d} f \mathrm{~d} g,
$$

where $f_{1}, f_{2} \in \mathcal{F}(M)$ and $\mathcal{F}(M)$ denotes the space of smooth functions on $M$. The $\mathbb{R}$-bilinear mapping (7) is skew-symmetric and satisfies the Jacobi identity. As is well-known, these key properties are central to the Hamiltonian formalism of classical mechanics developed for finitedimensional systems. The most remarkable observation that has been made independently in [11] and [7] is that the differential operator $P_{0}$ appearing in formula (2) can be used to define a Poisson bracket as well. Thus, in complete analogy with (7) one defines:

$$
\left\{F_{1}, F_{2}\right\}_{0}:=\int \frac{\delta F_{1}}{\delta u(x)} \frac{\partial}{\partial x} \frac{\delta F_{2}}{\delta u(x)} \mathrm{d} x .
$$

Remarkably, the bracket defined by (8) is also skew-symmetric and satisfies the Jacobi identity (see [16] for the proof). Equation (1) is rich in conserved quantities, three of which are classical:

$$
\begin{aligned}
& F_{0}=\int \frac{u}{2} \mathrm{~d} x, \\
& F_{1}=\int \frac{u^{2}}{2} \mathrm{~d} x, \\
& H_{0}=F_{2}=\int\left(u^{3}-\frac{u_{x}^{2}}{2}\right) \mathrm{d} x .
\end{aligned}
$$

In view of formula (5), their corresponding gradients are given by

$$
\begin{aligned}
G_{0} & =\frac{\delta F_{0}}{\delta u(x)}=\frac{1}{2}, \\
G_{1} & =\frac{\delta F_{1}}{\delta u(x)}=u, \\
G_{2} & =\frac{\delta H_{0}}{\delta u(x)}=\frac{\delta F_{2}}{\delta u(x)}=3 u^{2}+u_{x x} .
\end{aligned}
$$

The following few "simple" formulas had a paramount impact on the development of a theory which had ramifications and echoes in many areas of mathematics, including differential geometry, the theory of Lie groups, and Hamiltonian mechanics to name a few. The first observation is that the representation of type (2) for the $\mathrm{KdV}$ equation (1) is not unique. For example, the operator

$$
P_{1}=\frac{\partial^{3}}{\partial x^{3}}+4 u \frac{\partial}{\partial x}+2 u_{x}
$$

can be used to define another Poisson bracket in much the same way as in (8):

$$
\left\{F_{1}, F_{2}\right\}_{1}:=\int \frac{\delta F_{1}}{\delta u(x)} P_{1} \frac{\delta F_{2}}{\delta u(x)} \mathrm{d} x .
$$




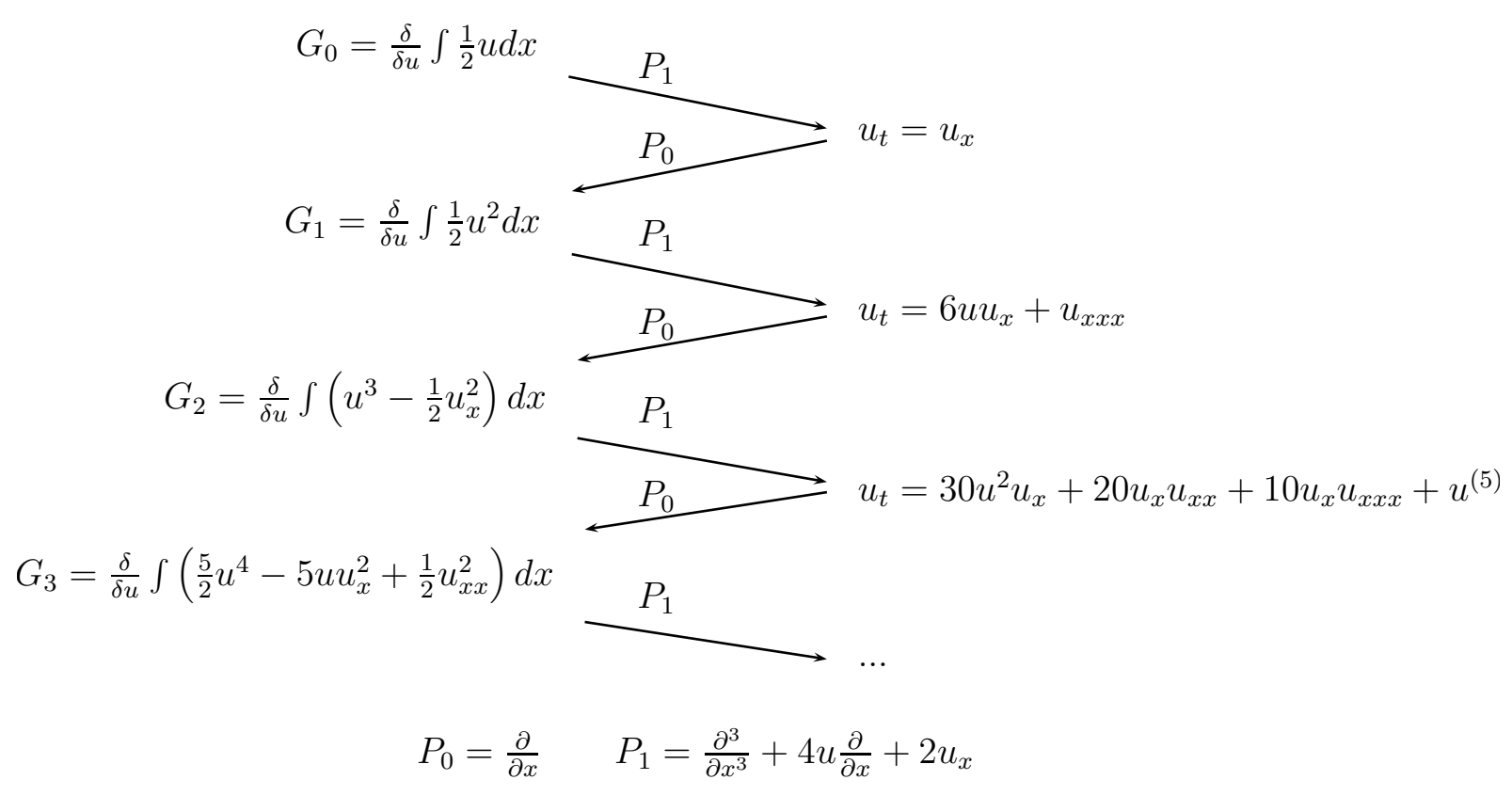

Figure 1. The Lenard recursion formula.

Remarkably, the Poisson bracket $\{\cdot, \cdot\}_{1}$ is also skew-symmetric and satisfies the Jacobi identity (see [24] for more details and proofs). Moreover, it can be matched with the corresponding Hamiltonian $H_{1}$, that together with (11) yields a formula analogous to (2):

$$
u_{t}=P_{1} \frac{\delta H_{1}}{\delta u(x)}
$$

where $H_{1}$ is given by

$$
H_{1}=F_{1}=\int \frac{u^{2}}{2} \mathrm{~d} x
$$

and the right hand side of (13) is the same as the right hand side of (1). Combining the formulas (2) and (13), one easily arrives at the following important formula which can be viewed as a precursor to the Lenard recursion formula:

$$
P_{1} G_{1}=\frac{\partial}{\partial x} G_{2}=P_{0} G_{2} .
$$

Note that in view of (8), (12), and (15), the functionals $F_{1}$ and $F_{2}$ (having the gradients $G_{1}$ and $G_{2}$ respectively) are in involution with respect to both $\{\cdot, \cdot\}_{0}$ and $\{\cdot, \cdot\}_{1}$ :

$$
\left\{F_{1}, F_{2}\right\}_{0}=\left\{F_{1}, F_{2}\right\}_{1}=0 .
$$

Furthermore, Lax proved in [16] the existence of more of these conserved functionals $F_{n}, n=$ $0,1,2, \ldots$ exhibiting the property (16). His proof is based on a generalization of the relation (15) which is nothing but the celebrated Lenard recursion formula:

$$
P_{1} G_{n}=\frac{\partial}{\partial x} G_{n+1}=P_{0} G_{n+1},
$$

where the $G_{n}$ 's are the gradients of the conserved functionals $F_{n}$ 's. It is easy to check that $G_{0}$, $G_{1}$ and $G_{2}$ given by (10) satisfy the relation (17). Furthermore, it easily follows from (17) that 
the functionals $F_{0}, F_{1}, F_{2}, \ldots$ corresponding to the gradients $G_{0}, G_{1}, G_{2}, \ldots$ generated via (17) are mutually in involution with respect to both Poisson brackets $\{\cdot, \cdot\}_{0}$ and $\{\cdot, \cdot\}_{1}$. In addition to the above, the Lenard recursion formula (17) has a number of important consequences, among which we single out the following two:

- The existence of two Hamiltonian representations for the KdV equation (1) gives rise to an infinite sequence of conserved functionals of (1).

- For every $n \geq 1$, the Lenard recursion formula (17) defines a higher order KdV equation, which has the same conserved quantities as the basic KdV equation (1). Therefore, the Lenard recursion formula leads to the KdV hierarchy.

We proceed with the following diagram (see Figure 1), which illustrates the properties and consequences of the Lenard recursion formula.

Shortly after this discovery concerning the KdV equation, it was shown by Magri [17] that the property of having two Hamiltonian representations was not a specific feature of the KdV equation alone, but rather a general property that could be found for other nonlinear PDEs with the same remarkable consequences. In his celebrated 1978 paper [17], Magri studied from this viewpoint, the Harry Dym and the modified KdV equations, thus developing a general scheme for studying these soliton equations as bi-Hamiltonian systems. A year later, in another fundamental paper, Gel'fand and Dorfman [13] extended these ideas to the field of finite-dimensional Hamiltonian systems. One cannot help noticing that Hamiltonian formalism originated in classical mechanics and was then applied to the study of soliton equations, while the bi-Hamiltonian formalism travelled in the opposite direction.

And the story began ...

\section{Andrew Lenard's story}

In this section we reproduce the story told by Andrew Lenard describing the events preceding and following the discovery of the Lenard recursion formula. In order to make the exposition clearer, we refer throughout the story to the corresponding references or the formulas presented in the previous section. Here is the story.

$$
* * *
$$

"Thank you for your communication. It is quite appropriate in the connection of your work, and I shall try to reply as best as I can.

In the earlier part of the 1960s, I was a scientific staff member of the Plasma Physics Laboratory (PPL) operated by Princeton University in conjunction with the Atomic Energy Commission. There, Martin Kruskal was a friend and colleague. He, together with his co-worker Norman Zabusky, discovered an astonishing phenomenon of the KdV differential equation, not until then noticed; namely, that in spite of its non-linear nature, certain wave solutions maintained their shapes unchanged after passing through a time interval of intense non-linear interaction ${ }^{2}$. This was followed by the discovery of a type of "linearization" of the problem by a functional transformation relating it to the 1-dimensional Schrödinger Equation ${ }^{3}$. In addition, first one and then several simply expressible constants of $\operatorname{motion}^{4}$ were found for the KdV evolution equation. Due to the combined work of Clifford Gardner, John Greene, and others, soon an infinite hierarchy of such constants of motion were generated ${ }^{5}$.

\footnotetext{
${ }^{2}$ The author refers to the results presented in [28].

${ }^{3}$ That is, the inverse scattering method [10].

${ }^{4}$ See $(9)$.

${ }^{5}$ See the references $[10,21,22]$.
} 
I left the PPL at this point to come to Indiana University. However, on a visit back to Princeton during the summer of 1967 (I believe) I went back to the PPL to see my old friends. It was there that something remarkable happened.

I arrived at coffee time in the afternoon. In the common room there were some blackboards. In front of one a crowd was gathered, centered around Kruskal, excitedly discussing something. I went up to ask what it was. They explained that another differential equation, similar to KdV but of higher order, was found, showing all those features of KdV I just described ${ }^{6}$. Someone wanted to know how one could systematically discover it, rather than just by lucky hit and miss. I heard Martin Kruskal shout at me: "There must be a method to generate many more, probably infinitely many such higher and higher order DE's, don't you think, Andrew?"

I asked for a yellow pad and pen, and went to sit down in a quiet corner to gather my thoughts. I was at that point particularly expert on generating functions as a means of summarizing information about infinite sequences in one mathematical construct. So naturally I tried this idea on the problem at hand, and it worked! It took me only fifteen minutes or so, and I could explain to the gathered friends how by means of a generating function an infinite hierarchy of KdV-like DE's could be generated, all of them having the same kind of behavior ${ }^{7}$.

This was greeted with admiration and satisfaction. I had my coffee and left.

Later, I saw that an article in the Comm. Appl. Math. (Courant Institute, NYU) by Gardner, Greene and Miura and perhaps others, had an appendix on my discovery ${ }^{8}$. I myself never published anything, nor concerned myself with the subject, then or since.

Several times during the intervening years I was surprised to hear my name being mentioned in connection with this, but actually much of it in connection with mathematics too high for me to appreciate. For instance, someone once told me that what I discovered was a dynamical system on a symplectic manifold with two different Hamiltonian structures ${ }^{9}$. And someone mentioned the "Lenard-Recursion Operator" ${ }^{10}$, and asked whether that was the same person as I.

Naturally, I am satisfied that I could make a contribution, even in such a fortuitous and judicious manner as I told you.

I hope this story will be satisfactory for you and answer your questions. By all means, feel free to share it with any like minded person if you care to. I don't mind it at all if the history of how "Lenard" became a concept in this area will be generally known.

Much good luck for your own studies, and sincerely yours:

Andrew Lenard

P.S. I recall that a mathematician at Dalhousie University (probably retired by now) whom I knew as a friend and colleague when he was at Indiana University during the early 1970s, is Peter Fillmore. Say hello to him for me if you see him."

$$
* * *
$$

\section{Acknowledgements}

We wish to thank Peter Olver for his careful reading of the manuscript and constructive comments. The first author (JP) would like to express his gratitude to fellow graduate students Caroline Adlam, Denis Falvey, Josh MacArthur, John Rumsey and Jin Yue for the stimulating atmosphere and useful discussions. The research was supported in part by a National Sciences and Engineering Research Council of Canada Discovery Grant (RGS).

\footnotetext{
${ }^{6}$ See Figure 1.

${ }^{7}$ See Figure 1.

${ }^{8}$ See the reference [12].

${ }^{9}$ See the formulas (2) and (13) as well as, for example, the references [13, 19, 24] for more details.

${ }^{10}$ See [24] for more details.
} 
[1] Andrà C., Degiovanni L., New examples of trihamiltonian structures linking different Lenard chains, in Proceedings of the International Conference on SPT2004 "Symmetry and Perturbation Theory" (May 30 June 6, 2004, Cala Gonone, Sardinia, Italy), Editors G. Gaeta, B. Prinari, S. Rauch-Wojciechowski and S. Terracini, World Scientific, 2005, 13-21.

[2] Błaszak M., Multi-Hamiltonian theory of dynamical systems, New York, Springer, 1998.

[3] Bogoyavlenskij O.I., Theory of tensor invariants of integrable Hamiltonian systems. I. Incompatible Poisson structures, Comm. Math. Phys., 1996, V.180, N 3, 529-586.

[4] Bolsinov A.V., Compatible Poisson brackets on Lie algebras and the completeness of families of functions in involution, Math. USSR-Izv., 1992, V.38, N 1, 69-90.

[5] Damianou P.A., Multiple Hamiltonian structures for Toda systems of types A-B-C, Regul. Chaotic Dyn., 2000, V.5, N 1, 17-32.

[6] Dickey L.A., Soliton equations and Hamiltonian systems, Singapore, World Scientific, 1991.

[7] Faddeev L.D., Zakharov V.E., The Korteweg-de Vries equation is a fully integrable Hamiltonian system, Funct. Anal. Appl., 1971, V.5, 18-27.

[8] Fernandes R.L., Completely integrable bi-Hamiltonian systems, J. Dynam. Diff. Equations, 1994, V.6, N 1, 63-69.

[9] Fokas A.S., Fuchssteiner B., On the structure of symplectic operators and hereditary symmetries, Lett. Nuovo Cimento, 1981, V.28, N 8, 299-303.

[10] Gardner C.S., Greene J.M., Kruskal M.D., Miura R.M., Method of solving the Korteweg-de Vries equation, Phys. Rev. Lett., 1967, V.19, 1095-1097.

[11] Gardner C.S., Korteweg-de Vries equation and generalizations. IV. The Korteweg-de Vries equations as a Hamiltonian system, J. Math. Phys., 1971, V.12, 1548-1551.

[12] Gardner C.S., Greene J.M., Kruskal M.D., Miura R.M., Korteweg-de Vries equation and generalizations. VI. Methods for exact solution, Comm. Pure Appl. Math., 1974, V.27, 97-133.

[13] Gel'fand I.M., Dorfman I.Ya., Hamiltonian operators and algebraic structures related to them, Funct. Anal. Appl., 1979, V.13, 248-262.

[14] Gel'fand I.M., Zakharevich I., Webs, Lenard schemes, and the local geometry of bi-Hamiltonian Toda and Lax structures, Selecta Math., 2000, V.6, N 2, 131-183.

[15] Kruskal M.D., Miura R.M., Gardner C.S., Zabusky N.J., Korteweg-de Vries equation and generalizations. V. Uniqueness and nonexistence of polynomial conservation laws, J. Math. Phys., 1970, V.11, 952-960.

[16] Lax P. D., Almost periodic solutions of the KdV equation, SIAM Review, 1976, V.18, N 3, 351-375.

[17] Magri F., A simple model of the integrable Hamiltonian equation, J. Math. Phys., 1978, V.19, $1156-1162$.

[18] Magri F., Lenard chains for classical integrable systems, Theoret. Mat. Fiz., 2003, V.137, N 3, 424-432 (in Russian).

[19] Magri F., Morosi C., A geometrical characterization of integrable Hamiltonian systems through the theory of Poisson-Nijenhuis manifolds, Quaderno, V.19, University of Milan, 1984.

[20] Magri F., Morosi C., Tondo G., Nijenhuis $G$-manifolds and Lenard bicomplexes: a new approach to KP systems, Comm. Math. Phys., 1988, V.115, N 3, 457-475.

[21] Miura R. M., Gardner C.S., Kruskal M.D., Korteweg-de Vries equation and generalizations. II. Existence of conservation laws and constants of motion, J. Math. Phys., 1968, V.9, 1204-1209.

[22] Miura R.M., Korteweg-de Vries equation and generalizations. I. A remarkable explicit non-linear transformation, J. Math. Phys., 1968, V.9, 1202-1204.

[23] Olver P.J., Evolution equations possessing infinitely many symmetries, J. Math. Phys., 1977, V.18, N 6, $1212-1215$

[24] Olver P.J., Applications of Lie groups to differential equations, 2nd ed., New York, Springer-Verlag, 1993.

[25] Smirnov R.G., Bi-Hamiltonian formalism: A constructive approach, Lett. Math. Phys., 1997, V.41, N 4, 333-347.

[26] Sergyeyev A., A simple way of making a Hamiltonian system into a bi-Hamiltonian one, Acta Appl. Math., 2004, V.83, N 1-2, 183-197.

[27] Su C.H., Gardner C.S., Korteweg-de Vries equation and generalizations. III. Derivation of the Korteweg-de Vries equation and Burgers equation, J. Math. Phys., 1969, V.10, 536-539.

[28] Zabusky N.J., Kruskal M.D., Interaction of "solitons" in a collisionless plasma and the recurrence of initial states, Phys. Rev. Lett., 1965, V.15, 240-243. 